\title{
Roles of Macrophage Subtypes in Bowel Anastomotic Healing and Anastomotic Leakage
}

\author{
Jinyao Shi (D), Zhouqiao Wu (D), Ziyu Li $(\mathbb{D}$, and Jiafu Ji \\ Gastrointestinal Cancer Center, Key Laboratory of Carcinogenesis and Translational Research (Ministry of Education), Peking \\ University Cancer Hospital \& Institute, No. 52 Fu-Cheng Road, Hai-Dian District, Beijing 100142, China \\ Correspondence should be addressed to Ziyu Li; ligregory@outlook.com
}

Received 28 July 2017; Revised 21 December 2017; Accepted 2 January 2018; Published 18 February 2018

Academic Editor: Zissis Chroneos

Copyright (c) 2018 Jinyao Shi et al. This is an open access article distributed under the Creative Commons Attribution License, which permits unrestricted use, distribution, and reproduction in any medium, provided the original work is properly cited.

\begin{abstract}
Macrophages play an important role in host defense, in addition to the powerful ability to phagocytose pathogens or foreign matters. They fulfill a variety of roles in immune regulation, wound healing, and tissue homeostasis preservation. Macrophages are characterized by high heterogeneity, which can polarize into at least two major extremes, M1-type macrophages (classical activation) which are normally derived from monocytes and M2-type macrophages (alternative activation) which are mostly those tissue-resident macrophages. Based on the wound healing process in skin, the previous studies have documented how these different subtypes of macrophages participate in tissue repair and remodeling, while the mechanism of macrophages in bowel anastomotic healing has not yet been established. This review summarizes the currently available evidence regarding the different roles of polarized macrophages in the physiological course of anastomotic healing and their pathological roles in anastomotic leakage, the most dangerous complication after gastrointestinal surgery.
\end{abstract}

\section{Introduction}

Macrophages are myeloid immune cells that play a central role in inflammation and host defense $[1,2]$. These cells are characterized by the powerful ability of phagocytosis and are credited with protecting the host from infection through a process so-called "innate immunity" [3]. In recent years, with the accumulation of evidence, macrophages have emerged as one of the most versatile cells. Their roles have shifted from immune effector cells which conduct host defense just as "trashmen" to predominant "directors" and "executors" for regulating inflammatory response, keeping tissue homeostasis, participating in wound healing and tissue remodeling [4].

Macrophages are actively involved in the wound healing process, while their role in a special surgical wound, also known as the anastomotic wound, has not yet been fully established. Anastomosis is constructed after removal of gastrointestinal tumor or bowel resection by surgeons to reconstruct the continuation of the gastrointestinal tract. Abnormal healing of anastomosis may develop into anastomotic leakage (AL), defined as luminal contents leaking from a surgical bowel connection [5]. It is the most dangerous complication after colorectal surgery [6-8], because it is responsible for up to $40 \%$ postoperative mortality rate, prolonged hospitalization, and an increase in the cost of healthcare due to the treatment of sepsis and the need for reoperation [9].

From a macroscopic point of view, the cause of $\mathrm{AL}$ mainly includes communication, infection, and healing disturbances [10]. However, a detailed mechanism on a cellular level is yet to be established due to the limited evidence. In this review, basing on heterogeneous populations of macrophages and their opposed tendencies of polarization, we tend to discuss the roles of different types of macrophages in an uneventful anastomotic healing and their pathological roles in anastomotic leakage.

1.1. Subtypes of Macrophages. Macrophages or mononuclear phagocytes had been long thought to originate from hematopoietic stem cells (HSCs). The prevailing dogma has stated that all macrophages derived from and were also replenished 
by monocytes [11]. However, macrophage family cells (cells of the mononuclear phagocyte system) manifest remarkable heterogeneity, in both their morphology and biological functions $[12,13]$. These recent data have challenged the long-held conception about "HSC-monocyte-macrophage." Evidence showed that tissue-resident macrophages like Kupffer cells of the liver, epidermal Langerhans cells of the skin, and microglia of the brain derived from a yolk sac and could persist in adult mice independent of HSCs [14-21]. Those tissue-resident macrophages can renew in situ, although they might also be replenished by blood monocytes in certain situations. In contrast to monocyte-derived macrophages which participate in an antibacterial process during acute inflammatory response, tissue-resident macrophages express different functional properties and play a central role in maintaining tissue architecture, function, and homeostasis [22-25], and their role in anastomotic healing is further discussed below.

The diversity and plasticity were recognized as hallmarks of macrophages, which contribute to their significant heterogeneity. In general, polarization of macrophages can be divided into two major extremes, that is, the classical activation which results in M1-type macrophages (M1) and the alternative activation which results in M2-type macrophages (M2). Those two types of macrophages perform diverse functional phenotypes in response to microenvironmental signals, like microbial products, damaged cells, and cytokines from activated lymphocytes. Specifically, ligands of Toll-like receptors (TLRs) and interferon- $\gamma$ (IFN- $\gamma$ ) can induce macrophages to polarize into M1-type macrophages; on the contrary, interleukin-4 (IL-4) and interleukin-13 (IL-13) induce macrophages to polarize into M2-type macrophages [26-28]. However, such explanation may not fully illustrate all different activation scenarios. Murray et al. proposed that there should be some other subtypes between M1 and M2 [29], including the M2a subgroup induced by IL- 4 and IL-13, the M2b subgroup activated by immune complexes (TLRs), and the M2c subgroup deactivated by glucocorticoids, transforming growth factor (TGF), or interleukin-10 (IL-10) [30, 31]. Moreover, it is also reported that there might be a supplementary subtype of M2 (M2d), which is elicited by TLR agonists and adenosine $[32,33]$. It seems that the polarization of macrophages should be viewed as a continued spectrum, on which, two types of macrophages (M1 and M2) occupied the opposite ends. Another classification of polarization proposed by Mosser and Edwards suggested that macrophages are activated to form three populations in charge of host defense, wound healing, and immune regulation, respectively [34]. The authors classified macrophages on the basis of their fundamental functions rather than of the stimuli. Matching with the previously discussed conception of "M1-M2" paradigm, most of monocyte-derived macrophages are classically activated and express the M1 phenotype, which exerts host defense; reversely, tissue-resident macrophages are mainly activated in the alternative pathway which expresses M2like characteristic and preserves tissue homeostasis and resolution of inflammation [21-23].
1.2. The Role of Polarized Macrophages in Physiological Anastomotic Healing. The wall of the alimentary tract contains four layers (i.e., mucosa, submucosa, muscularis propria, and serosa). For a classic end-to-end inverted bowel anastomosis, apposition of the serosa vanishes the gap between the two ends of the gastrointestinal tract, providing a barrier that insulates the sterile abdominal cavity from luminal contents and bacteria; moreover, this layer is important in providing a matrix for fibroblasts [35]. The submucosa consists of blood vessels, lymphatics, and nerve fibers; this layer is the source of fibroblasts that become active after gastrointestinal surgery and start to deposit collagen. The stapled or sutured collagen fibers in this layer provide most of the tensile strength of anastomosis [36]; hence, the submucosa is of great importance in anastomotic healing. The mucosal layer also plays a role in maintaining homeostasis to allow the healing process. A pool of macrophages in the gastrointestinal mucosa is the largest pool of tissue macrophages in the body, and a long-lasting macrophage absence or dysfunction impairs anastomotic healing $[37,38]$.

Tissue repair and healing after injury have been studied for centuries but remain understood to a limited object, that is, skin. Different from that, healing of the gastrointestinal tract is anatomically obscured from inspection, only allowing the surgeon to judge the success of the operation only on the patient's parameters of general wellbeing [36]. There are some differences between the skin and anastomotic healing including anatomy (e.g., no equivalent anatomic component of the serosa in cutaneous tissues) and collagen and collagenase activity [39]. However, classical response to injury occurs in all organs and tissues. The physiological course of anastomotic healing can also be divided into three overlapping but distinct stages, which include inflammation, new tissue formation, and remodeling (Figure 1) [40,41].

1.2.1. Inflammation. In addition to infection of diverse microbial factors, injuries or traumas such as surgical strike can also lead to a non-pathogen-associated inflammation, which can be further divided into the early inflammatory response and the late one [42]. In the early inflammatory phase, neutrophils are recruited from circulating blood to local wounding tissue (the anastomotic area) at first. Those recruited polymorphonuclear cells remove the local foreign particles or bacteria and then undergo apoptosis or necrosis. After that, monocytes are recruited and differentiate into macrophages which are highly phagocytic. They phagocytose impaired neutrophils and other tissue debris to protect from further tissue damage. During this phase, in response to pathogen-associated modifying patterns (PAMPs) in a contaminative circumstance or damage-associated modifying patterns (DAMPs) in a sterile circumstance, macrophages are classically activated and express the M1 phenotype [43-45]. M1 macrophages release high concentrations of proinflammatory cytokines such as tumor necrosis factor- $\alpha$ (TNF- $\alpha$ ), interleukin-1 $\beta$ (IL-1 $\beta$ ), interleukin-6 (IL-6), and interleukin-12 (IL-12); protease; and reactive oxygen species (ROS) [34], all of which are thought to be important for 


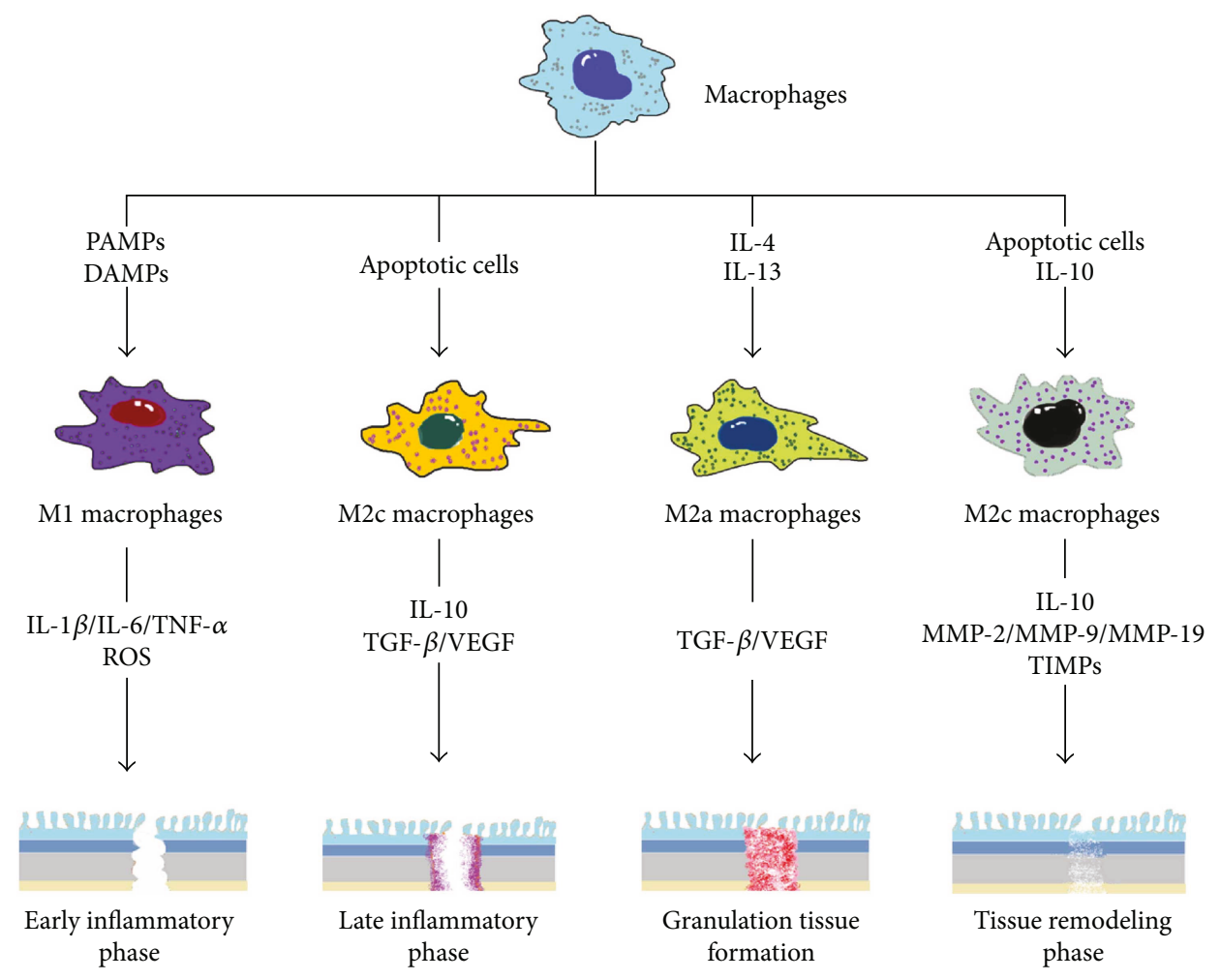

FIGURE 1: Polarization of macrophages in normal healing of anastomosis. Inactivated macrophages can be stimulated by various stimuli (e.g., PAMP, DAMP/IL-4, and IL-13/apoptotic cell) and polarize into M1- or M2- (M2a, M2c) type macrophages during different phases of normal anastomotic healing. Differentiated macrophages express a variety of cytokines (e.g., IL-1 $\beta$, IL-6, IL-10, and TNF- $\alpha$ ), growth factors (e.g., VEGF), and enzymes (MMPs). These biochemical substances acting upon tissues contribute to tissue repair and remodeling. PAMP: pathogen-associated modifying patterns; DAMP: damage-associated modifying patterns; IL: interleukin; TNF- $\alpha$ : tumor necrosis factor- $\alpha$; VEGF: vascular endothelial growth factor; MMPs: matrix metalloproteinases.

microbial killing and proinflammatory response [13]. M1 macrophages can also produce collagenase, a high-activity enzyme that causes collagen degradation that results in low anastomotic strength early after the formation of an anastomosis [46]. In the late inflammation phase, with excessive phagocytosis of apoptotic neutrophils, engagement of $\beta 2$ integrins on macrophages by apoptotic neutrophils activates macrophages to express anti-inflammatory mediator transforming growth factor (TGF) [47]. In contrast, the production of proinflammatory cytokines like TNF- $\alpha$ and IL- $1 \beta$ was inhibited $[48,49]$. Thus, the phenotype of macrophages switches from proinflammatory M1-like to anti-inflammatory M2-like. These macrophages produce cytokines such as IL-10 and lay the foundation for new tissue formation by secreting other growth factors such as vascular endothelial growth factor (VEGF) [50, 51]. Because macrophages stimulated with IL-10, TGF, or glucocorticoids in vitro polarize into the M2c subtype that shares similarities with anti-inflammatory macrophages $[30,52-58]$, it suggests that anti-inflammatory macrophages belong to M2c-type macrophages and are able to amplify their anti-inflammatory response by secreting IL-10 and TGF in a feedforward loop. In addition, anti-inflammatory and regenerative capacities of anti-inflammatory macrophages were shown to be entirely IL-10-dependent in sterile environments, for example, in surgical wound [59].
1.2.2. New Tissue Formation. In this phase, macrophages resident in tissue or recruited from peripheral blood, known as profibrotic macrophages, generate various growth factors such as TGF, platelet-derived growth factor (PDGF), fibroblast growth factor-2, or insulin-like growth factor-1 [60]. Among them, TGF is a profibrotic cytokine that exerts on fibroblasts and activates them to differentiate into myofibroblasts in wound tissue. Myofibroblasts produce a mass of extracellular matrix (ECM) components including collagen and fibronectin to fill up the tissue defect. For the gastrointestinal tract, collagen can also be produced by smooth muscle cells [61]. Collagen subtypes in the gastrointestinal tract are collagens I, III, and V, compared to solely collagen I and III in the skin [62]. By efficient contractile forces from myofibroblasts, fractured wound tissue can be bound together and rebuild their integrity [63]. Meanwhile, profibrotic macrophages and activated fibroblasts release proangiogenic factors like VEGF, which elicit endothelial progenitor cells crawling towards wound tissue, to promote new vessel formation (angiogenesis). Invasion of the capillary increases the blood supply to local tissues and facilitates anastomotic healing. Furthermore, studies of the healing colonic mucosa of rabbits after experimental excision showed that an abundance of mesenchymal cells in the healing intestinal muscle layers accompanies capillary invasion; these cells can differentiate into smooth muscle cells and histiocytes, which are thought 
to be responsible for the reestablishment of smooth muscle tissue $[64,65]$. Profibrotic macrophages, myofibroblasts, and neovessels all together constitute granulation tissue, the most important fundamental compartment in the normal course of wound healing [40, 41]. These profibrotic macrophages are functionally classified as M2a-like macrophages because they can be induced in vitro by IL-4 and IL-13 [23, 30]. However, it is not clear whether antiinflammatory and profibrotic macrophages can be clearly distinguished in vivo, and it appears that macrophage plasticity creates a mixture or continuous variant shifts during wound healing [50].

1.2.3. Remodeling. Remodeling of anastomosis is a dynamic process of maturation within healed tissue that is based on a balance between ECM deposition and breakdown and tissue remodeling [66, 67]. A part of tissue-resident macrophages termed as fibrolytic macrophages is critical for maintaining this dynamic balance. They produce matrix metalloproteinases like matrix metalloproteinase-2 (MMP2), matrix metalloproteinase-9 (MMP9), matrix metalloproteinase-12 (MMP12), and matrix metalloproteinase-19 (MMP19) $[42,68]$, to degrade matrix macromolecules, that is, collagen, one of the most important components of ECM. The submucosa is a strength layer of the gastrointestinal tract and made predominantly of collagen, and remodeling of this layer predominates the strength of the anastomosis. Depending on MMPs secreted by fibrolytic macrophages, initially deposited collagen fibers are rearranged and cross-linked, remodeled from type III collagen to type I collagen; the latter one is much stronger. Besides, fibrolytic macrophages also regulate the degradation by synthesizing the tissue inhibitor of metalloproteinases (TIMPs), which can inhibit the activities of MMPs. Furthermore, fibrolytic macrophages are responsible for the induction of fibroblast apoptosis, subsequent removal of apoptotic cells, and suppression of further inflammation via IL-10 release [60]. Fibrolytic macrophages are proposed to be classified as M2c-like macrophages which can be elicited in vitro by apoptotic cells and IL-10 [69, 70].

Thus, macrophages participate in whole physiological courses of anastomotic healing. Among the three main phases of tissue repair, macrophages express different phenotypes during different stages. There are at least four kinds of macrophages in a condition of normal tissue repair: (1) proinflammatory macrophages, (2) anti-inflammatory macrophages, (3) profibrotic macrophages, and (4) fibrolytic macrophages. If we sort out those four kinds of macrophages according to "M1-M2" paradigm, proinflammatory and profibrotic macrophages may, respectively, correspond to M1-type and M2a-type macrophages. Meanwhile, both anti-inflammatory and fibrolytic macrophages probably belong to M2c-type macrophages $[42,59]$.

1.3. Roles of Macrophages in Anastomotic Leakage. As we previously discussed in our review, occurrence of AL mainly contains three factors: communication, infection, and healing disturbances. Communication means defect of the alimentary tract in the anastomotic region that connects the gastrointestinal lumina and abdominal cavity. Infection indicates anastomotic site bacterial infection. Healing disturbances include all substances that disturb a normal healing process such as hypoxia or inflammation. These three factors actively interact with each other: one factor takes place, and a responsive chain that consists of all factors will be initiated, eventually leading to AL. For example, infection provokes inflammatory response at the anastomotic site, which impairs collagen deposition [71, 72], then interferes with the normal healing process, and leads to a communication between the intra- and extraluminal gastrointestinal walls. On the contrary, communication allows the bowel content (including bacteria) to dislocate into the abdominal cavity, causes intra-abdominal infection, and afterwards delays anastomosis healing. Clinically, communication in some extent is regarded as a macroscopic clinical outcome, while infection and healing disturbances are durative biological processes. For AL, macrophages are mainly involved in the latter two mechanisms, which is also observed in other poorly healed wounds [73-77].

Anastomotic infection may be caused by anastomotic dehiscence (intestinal contents leak to the sterile abdominal cavity) or pre-/intraoperative contamination. Regardless of the cause of infection, in the contaminative infective environment, macrophages polarize into the M1 type as mentioned above. However, instead of supporting resistance to intracellular bacteria and controlling the acute phase of infection, an excessive or prolonged M1 program is deleterious for patients, as demonstrated in acute infections with Escherichia coli [78]. E. coli as a resident flora of the gut can induce a typical M1 profile through the recognition of lipopolysaccharides (LPS) by TLR4 [79, 80]. Classical activated M1-type macrophages upregulate the expression of inducible nitric oxide synthase (iNOS), which is responsible for the generation of nitric oxide (NO). NO was first identified to mediate arterial vasodilatation [81-83] and then was found to have a role in host defense against pathogens [84, 85]. Moreover, a prominent role has been described for $\mathrm{NO}$ in collagen deposition, fibrosis, and scar formation [71, 72, 86, 87]. High levels of wound NO, as in infection or inflammation, severely impair wound collagen synthesis [88]. Decreased deposition of collagen seriously weakens the anastomotic strength, which may lead to the failure of anastomotic healing. Therefore, improper M1 polarization of macrophages in bacterial infection of the abdominal cavity contributes to the occurrence of AL.

The role of macrophages in leakage with healing disturbances is more complicated. During a normal condition, tissue repair initiates from clearance of tissue debris and dead cells, efficiently phagocytosing those "tissue rubbish" by macrophages, and is critical for timely resolution of inflammation and successful healing. Nevertheless, for those patients complicated with diabetes mellitus, advancing in years, or undergone chemotherapy, the ability of macrophages to phagocytose is severely influenced, which directly leads to an accumulation of apoptotic or necrotic cells at the anastomosis site. This accumulation of dead cells prolongs the inflammatory phase, disturbs the healing process, and compromises the resolution of inflammation [73, 74]. 


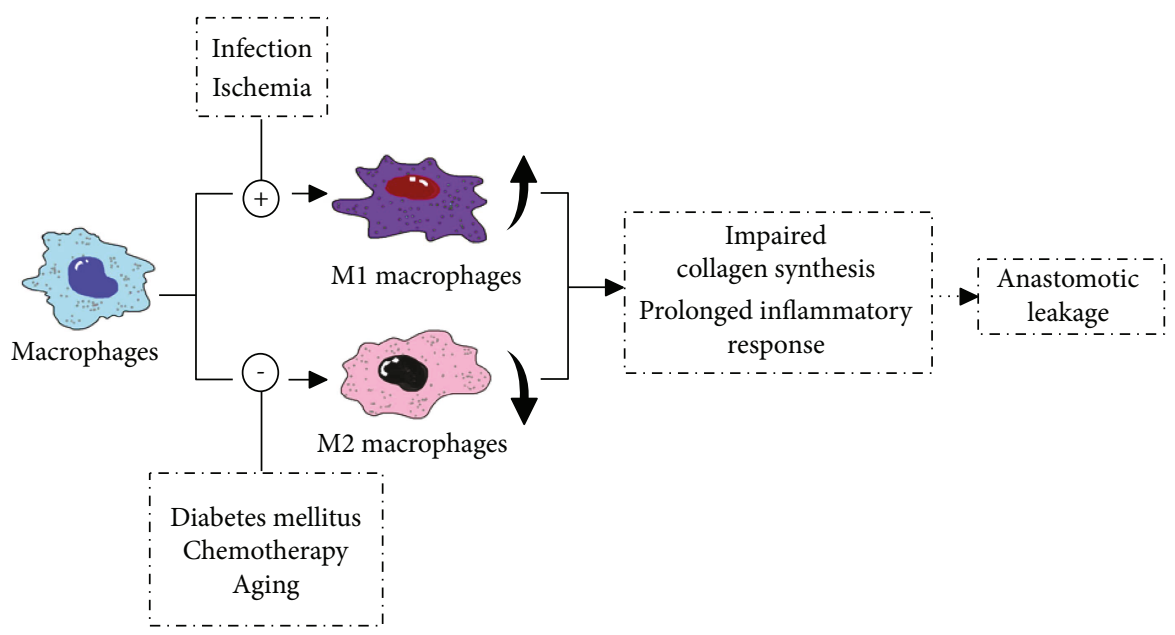

FIgURE 2: Imbalance of polarized macrophages contributes to the occurrence of anastomotic leakage. In some pathological conditions (infection, ischemia, diabetes mellitus, etc.), macrophages are abnormally activated into M1-type macrophages or inhibited to express the M2-type phenotype, which leads to long-time inflammatory response in the anastomotic site and influences collagen deposition and tissue repair; all of those are thought to be associated with anastomotic leakage.

Other disturbances such as ischemia or anastomotic hypoxia severely compromise the anastomotic healing $[89,90]$. At a cellular level, exposing macrophages to an anoxic environment leads to the expression of proinflammatory cytokines like IL- $1 \beta$ and TNF- $\alpha$ and cytotoxic mediators like NO [91-93], which indicates that hypoxia can promote macrophages to polarize into the M1 phenotype. Excessively activated M1 macrophages sustain proinflammatory response and obstruct subsequent steps of the repair process that influences proper healing and remodeling of anastomosis [94-96], and the relevant mechanism is described above.

Based on the available evidence, it seems that classical activated macrophages which express the M1 phenotype are responsible for the pathological process of defective anastomotic healing, whereas alternative activated macrophages which express the M2 phenotype play a critical role in inflammation resolution and successful tissue repair (Figure 2). Although M1-type macrophages participate in the early phase of normal wound healing, the programmed transformation of their polarized orientation from M1 to M2 lays the foundation of transient inflammatory response and the following tissue regeneration.

\section{Conclusion}

Macrophages are the most versatile immune cells and possess significant plasticity and heterogeneity. Macrophages can polarize into two main extremes and express corresponding phenotypes (M1 and M2). As polarization is the premise for macrophages to exert their diverse biological functions, different polarized macrophages play different roles in the physiological process of anastomotic healing and pathogenesis of AL. Reacquainting AL in the perspective of macrophages contributes to the exploration of new diagnostic tools and therapeutic targets. For example, in different recovery phases after anastomosis construction, the spectrum of cytokines and inflammatory mediators such as IL- $1 \beta$, IL- 6 , IL-10, IL-12, TNF- $\alpha$, ROS, and NO, which are secreted by macrophages, may appear an alteration. Moreover, the level of these substances could indirectly reflect the situation of an anastomosis. An abnormal fluctuation of these substances probably indicates disorder and defection of anastomosis healing, which can be regarded as premonition of AL. Because M1-type macrophages show a stimulating effect on AL and M2 macrophages are essential for anastomosis healing, regulation of M1/M2 polarization may find its therapeutic roles in the treatment of $\mathrm{AL}$ in the future.

\section{Conflicts of Interest}

The authors declare that there is no conflict of interest regarding the publication of this article.

\section{Authors' Contributions}

Jinyao Shi and Zhouqiao $\mathrm{Wu}$ are the first authors.

\section{Acknowledgments}

This paper is supported by the Beijing Municipal Science and Technology Commission (nos. D141100000414004 and Z151100004015070) and Beijing Municipal Commission of Health and Family Planning (no. 2014-1-2151).

\section{References}

[1] C. Varol, A. Mildner, and S. Jung, "Macrophages: development and tissue specialization," Annual Review of Immunology, vol. 33, no. 1, pp. 643-675, 2015.

[2] S. Gordon and F. O. Martinez, "Alternative activation of macrophages: mechanism and functions," Immunity, vol. 32, no. 5 , pp. 593-604, 2010.

[3] C. Nathan, "Metchnikoff's legacy in 2008," Nature Immunology, vol. 9, no. 7, pp. 695-698, 2008.

[4] E. S. Edholm, K. H. Rhoo, and J. Robert, "Evolutionary Aspects of Macrophages Polarization," in Macrophage Origin, 
Functions and Bio Intervention, M. Kloc, Ed., Springer International Publishing AG, Switzerland, 2017.

[5] S. W. Leichtle, N. J. Mouawad, K. B. Welch, R. M. Lampman, and R. K. Cleary, "Risk factors for anastomotic leakage after colectomy," Diseases of the Colon and Rectum, vol. 55, no. 5, pp. 569-575, 2012.

[6] B. C. Paun, S. Cassie, A. R. MacLean, E. Dixon, and W. D. Buie, "Postoperative complications following surgery for rectal cancer," Annals of Surgery, vol. 251, no. 5, pp. 807-818, 2010.

[7] C. Y. Kang, W. J. Halabi, O. O. Chaudhry et al., "Risk factors for anastomotic leakage after anterior resection for rectal cancer," JAMA Surgery, vol. 148, no. 1, pp. 65-71, 2013.

[8] C. S. McArdle, D. C. McMillan, and D. J. Hole, "Impact of anastomotic leakage on long-term survival of patients undergoing curative resection for colorectal cancer," The British Journal of Surgery, vol. 92, no. 9, pp. 1150-1154, 2005.

[9] M. A. Boccola, P. G. Buettner, W. M. Rozen et al., "Risk factors and outcomes for anastomotic leakage in colorectal surgery: a single-institution analysis of 1576 patients," World Journal of Surgery, vol. 35, no. 1, pp. 186-195, 2011.

[10] C. L. Sparreboom, Z. Q. Wu, J. F. Ji, and J. F. Lange, "Integrated approach to colorectal anastomotic leakage: communication, infection and healing disturbances," World Journal of Gastroenterology, vol. 22, no. 32, pp. 7226-7235, 2016.

[11] R. van Furth, Z. A. Cohn, J. G. Hirsch, J. H. Humphrey, W. G. Spector, and H. L. Langevoort, "The mononuclear phagocyte system: a new classification of macrophages, monocytes, and their precursor cells," Bulletin of the World Health Organization, vol. 46, no. 6, pp. 845-852, 1972.

[12] S. Gordon and P. R. Taylor, "Monocyte and macrophage heterogeneity," Nature Reviews Immunology, vol. 5, no. 12, pp. 953-964, 2005.

[13] S. Gordon, "Alternative activation of macrophages," Nature Reviews Immunology, vol. 3, no. 1, pp. 23-35, 2003.

[14] C. Schulz, E. G. Perdiguero, L. Chorro et al., "A lineage of myeloid cells independent of Myb and hematopoietic stem cells," Science, vol. 336, no. 6077, pp. 86-90, 2012.

[15] M. Merad, M. G. Manz, H. Karsunky et al., "Langerhans cells renew in the skin throughout life under steady-state conditions," Nature Immunology, vol. 3, no. 12, pp. 1135-1141, 2002.

[16] F. Ginhoux, M. Greter, M. Leboeuf et al., "Fate mapping analysis reveals that adult microglia derive from primitive macrophages," Science, vol. 330, no. 6005, pp. 841-845, 2010.

[17] D. Hashimoto, A. Chow, C. Noizat et al., "Tissue-resident macrophages self-maintain locally throughout adult life with minimal contribution from circulating monocytes," Immunity, vol. 38, no. 4, pp. 792-804, 2013.

[18] S. Yona, K. W. Kim, Y. Wolf et al., "Fate mapping reveals origins and dynamics of monocytes and tissue macrophages under homeostasis," Immunity, vol. 38, no. 1, pp. 79-91, 2013.

[19] S. Epelman, K. J. Lavine, A. E. Beaudin et al., "Embryonic and adult-derived resident cardiac macrophages are maintained through distinct mechanisms at steady state and during inflammation," Immunity, vol. 40, no. 1, pp. 91-104, 2014.

[20] E. Gomez Perdiguero, K. Klapproth, C. Schulz et al., "Tissueresident macrophages originate from yolk-sac-derived erythro-myeloid progenitors," Nature, vol. 518, no. 7540, pp. 547-551, 2015.
[21] L. C. Davies, S. J. Jenkins, J. E. Allen, and P. R. Taylor, “Tissueresident macrophages," Nature Immunology, vol. 14, no. 10, pp. 986-995, 2013.

[22] A. Mantovani, A. Sica, and M. Locati, "Macrophage polarization comes of age," Immunity, vol. 23, no. 4, pp. 344-346, 2005.

[23] A. Mantovani, S. K. Biswas, M. R. Galdiero, A. Sica, and M. Locati, "Macrophage plasticity and polarization in tissue repair and remodelling," The Journal of Pathology, vol. 229, no. 2, pp. 176-185, 2013.

[24] E. G. Perdiguero and F. Geissmann, "The development and maintenance of resident macrophages," Nature Immunology, vol. 17, no. 1, pp. 2-8, 2016.

[25] G. Hoeffel, J. Chen, Y. Lavin et al., "C-Myb ${ }^{+}$erythro-myeloid progenitor-derived fetal monocytes give rise to adult tissueresident macrophages," Immunity, vol. 42, no. 4, pp. 665-678, 2015.

[26] A. Mantovani, S. Sozzani, M. Locati, P. Allavena, and A. Sica, "Macrophage polarization: tumor-associated macrophages as a paradigm for polarized M2 mononuclear phagocytes," Trends in Immunology, vol. 23, no. 11, pp. 549-555, 2002.

[27] S. K. Biswas and A. Mantovani, "Macrophage plasticity and interaction with lymphocyte subsets: cancer as a paradigm," Nature Immunology, vol. 11, no. 10, pp. 889-896, 2010.

[28] A. Sica and V. Bronte, "Altered macrophage differentiation and immune dysfunction in tumor development," The Journal of Clinical Investigation, vol. 117, no. 5, pp. 1155-1166, 2007.

[29] P. J. Murray, J. E. Allen, S. K. Biswas et al., "Macrophage activation and polarization: nomenclature and experimental guidelines," Immunity, vol. 41, no. 1, pp. 14-20, 2014.

[30] A. Mantovani, A. Sica, S. Sozzani, P. Allavena, A. Vecchi, and M. Locati, "The chemokine system in diverse forms of macrophage activation and polarization," Trends in Immunology, vol. 25, no. 12, pp. 677-686, 2004.

[31] F. O. Martinez and S. Gordon, "The M1 and M2 paradigm of macrophage activation: time for reassessment," F1000Prime Reports, vol. 6, 2014.

[32] S. Colin, G. Chinetti-Gbaguidi, and B. Staels, "Macrophage phenotypes in atherosclerosis," Immunological Reviews, vol. 262, no. 1, pp. 153-166, 2014.

[33] C. J. Ferrante and S. J. Leibovich, "Regulation of macrophage polarization and wound healing," Advances in Wound Care, vol. 1, no. 1, pp. 10-16, 2012.

[34] D. M. Mosser and J. P. Edwards, "Exploring the full spectrum of macrophage activation," Nature Reviews Immunology, vol. 8, no. 12, pp. 958-969, 2008.

[35] F. Daams, K. Monkhorst, J. van den Broek, J. C. Slieker, J. Jeekel, and J. F. Lange, "Local ischaemia does not influence anastomotic healing: an experimental study," European Surgical Research, vol. 50, no. 1, pp. 24-31, 2013.

[36] F. J. Thornton and A. Barbul, "Healing in the gastrointestinal tract," The Surgical Clinics of North America, vol. 77, no. 3, pp. 549-573, 1997.

[37] D. Pantelis, A. Beissel, P. Kahl et al., "Colonic anastomotic healing in the context of altered macrophage function and endotoxemia," International Journal of Colorectal Disease, vol. 26, no. 6, pp. 737-746, 2011.

[38] P. D. Smith, L. E. Smythies, R. Shen, T. Greenwell-Wild, M. Gliozzi, and S. M. Wahl, "Intestinal macrophages and response to microbial encroachment," Mucosal Immunology, vol. 4 , no. 1 , pp. 31-42, 2011. 
[39] S. K. Thompson, E. Y. Chang, and B. A. Jobe, "Clinical review: healing in gastrointestinal anastomoses, part I," Microsurgery, vol. 26, no. 3, pp. 131-136, 2006.

[40] G. C. Gurtner, S. Werner, Y. Barrandon, and M. T. Longaker, "Wound repair and regeneration," Nature, vol. 453, no. 7193, pp. 314-321, 2008.

[41] A. J. Singer and R. A. Clark, "Cutaneous wound healing," The New England Journal of Medicine, vol. 341, no. 10, pp. 738-746, 1999.

[42] A. Sindrilaru and K. Scharffetter-Kochanek, "Disclosure of the culprits: macrophages-versatile regulators of wound healing," Advances in Wound Care, vol. 2, no. 7, pp. 357-368, 2013.

[43] C. A. Janeway Jr and R. Medzhitov, "Innate immune recognition," Annual Review of Immunology, vol. 20, no. 1, pp. 197-216, 2002.

[44] X. Zhang and D. M. Mosser, "Macrophage activation by endogenous danger signals," The Journal of Pathology, vol. 214, no. 2, pp. 161-178, 2008.

[45] T. J. Koh and L. A. DiPietro, "Inflammation and wound healing: the role of the macrophage," Expert Reviews in Molecular Medicine, vol. 13, article e23, 2011.

[46] S. Munireddy, S. L. Kavalukas, and A. Barbul, "Intraabdominal healing: gastrointestinal tract and adhesions," The Surgical Clinics of North America, vol. 90, no. 6, pp. 1227-1236, 2010.

[47] T. Peters, A. Sindrilaru, B. Hinz et al., "Wound-healing defect of CD18-/- mice due to a decrease in TGF- $\beta 1$ and myofibroblast differentiation," The EMBO Journal, vol. 24, no. 19, pp. 3400-3410, 2005.

[48] V. A. Fadok, D. L. Bratton, A. Konowal, P. W. Freed, J. Y. Westcott, and P. M. Henson, "Macrophages that have ingested apoptotic cells in vitro inhibit proinflammatory cytokine production through autocrine/paracrine mechanisms involving TGF-beta, PGE2, and PAF," The Journal of Clinical Investigation, vol. 101, no. 4, pp. 890-898, 1998.

[49] M. L. Huynh, V. A. Fadok, and P. M. Henson, "Phosphatidylserine-dependent ingestion of apoptotic cells promotes TGF$\beta 1$ secretion and the resolution of inflammation," The Journal of Clinical Investigation, vol. 109, no. 1, pp. 41-50, 2002.

[50] S. J. Galli, N. Borregaard, and T. A. Wynn, "Phenotypic and functional plasticity of cells of innate immunity: macrophages, mast cells and neutrophils," Nature Immunology, vol. 12, no. 11, pp. 1035-1044, 2011.

[51] M. Lucas, L. M. Stuart, J. Savill, and A. Lacy-Hulbert, "Apoptotic cells and innate immune stimuli combine to regulate macrophage cytokine secretion," Journal of Immunology, vol. 171, no. 5, pp. 2610-2615, 2003.

[52] Q. Cao, Y. Wang, D. Zheng et al., "IL-10/TGF- $\beta$-modified macrophages induce regulatory $\mathrm{T}$ cells and protect against adriamycin nephrosis," Journal of the American Society of Nephrology, vol. 21, no. 6, pp. 933-942, 2010.

[53] D. C. Kluth, C. V. Ainslie, W. P. Pearce et al., "Macrophages transfected with adenovirus to express IL-4 reduce inflammation in experimental glomerulonephritis," Journal of Immunology, vol. 166, no. 7, pp. 4728-4736, 2001.

[54] Y. Wang, Y. P. Wang, G. Zheng et al., "Ex vivo programmed macrophages ameliorate experimental chronic inflammatory renal disease," Kidney International, vol. 72, no. 3, pp. 290299, 2007.

[55] D. Zheng, Y. Wang, Q. Cao et al., "Transfused macrophages ameliorate pancreatic and renal injury in murine diabetes mellitus," Nephron Experimental Nephrology, vol. 118, no. 4, pp. e87-e99, 2011.

[56] K. Barczyk, J. Ehrchen, K. Tenbrock et al., "Glucocorticoids promote survival of anti-inflammatory macrophages via stimulation of adenosine receptor A3," Blood, vol. 116, no. 3, pp. 446-455, 2010.

[57] R. Bertalan, A. Patocs, B. Vasarhelyi et al., "Association between birth weight in preterm neonates and the BclI polymorphism of the glucocorticoid receptor gene," The Journal of Steroid Biochemistry and Molecular Biology, vol. 111, no. 1-2, pp. 91-94, 2008.

[58] T. Roszer, "Understanding the mysterious M2 macrophage through activation markers and effector mechanisms," Mediators of Inflammation, vol. 2015, Article ID 816460, 16 pages, 2015.

[59] M. Weidenbusch and H. J. Anders, “Tissue microenvironments define and get reinforced by macrophage phenotypes in homeostasis or during inflammation, repair and fibrosis," Journal of Innate Immunity, vol. 4, no. 5-6, pp. 463-477, 2012.

[60] J. S. Duffield, M. Lupher, V. J. Thannickal, and T. A. Wynn, "Host responses in tissue repair and fibrosis," Annual Review of Pathology, vol. 8, no. 1, pp. 241-276, 2013.

[61] M. F. Graham, D. E. Drucker, R. F. Diegelmann, and C. O. Elson, "Collagen synthesis by human intestinal smooth muscle cells in culture," Gastroenterology, vol. 92, no. 2, pp. 400-405, 1987.

[62] J. W. Bosmans, A. C. Jongen, N. D. Bouvy, and J. P. Derikx, "Colorectal anastomotic healing: why the biological processes that lead to anastomotic leakage should be revealed prior to conducting intervention studies," BMC Gastroenterology, vol. 15, no. 1, p. 180, 2015.

[63] B. Hinz, S. H. Phan, V. J. Thannickal et al., "Recent developments in myofibroblast biology: paradigms for connective tissue remodeling," The American Journal of Pathology, vol. 180, no. 4, pp. 1340-1355, 2012.

[64] N. Mori, Y. Doi, K. Hara, M. Yoshizuka, K. Ohsato, and S. Fujimoto, "Role of multipotent fibroblasts in the healing colonic mucosa of rabbits. Ultrastructural and immunocytochemical study," Histology and Histopathology, vol. 7, no. 4, pp. 583-590, 1992.

[65] F. J. Thornton, A. Barbul, and FACS, "Healing in the gastrointestinal tract," Surgical Clinics of North America, vol. 77, no. 3, pp. 549-573, 1997.

[66] M. P. Welch, G. F. Odland, and R. A. Clark, “Temporal relationships of F-actin bundle formation, collagen and fibronectin matrix assembly, and fibronectin receptor expression to wound contraction," The Journal of Cell Biology, vol. 110, no. 1, pp. 133-145, 1990.

[67] J. B. Lundy, "A primer on wound healing in colorectal surgery in the age of bioprosthetic materials," Clinics in Colon and Rectal Surgery, vol. 27, no. 4, pp. 125-133, 2014.

[68] S. D. Shapiro, D. K. Kobayashi, and T. J. Ley, "Cloning and characterization of a unique elastolytic metalloproteinase produced by human alveolar macrophages," The Journal of Biological Chemistry, vol. 268, no. 32, pp. 23824-23829, 1993.

[69] H. J. Anders and M. Ryu, "Renal microenvironments and macrophage phenotypes determine progression or resolution of renal inflammation and fibrosis," Kidney International, vol. 80, no. 9, pp. 915-925, 2011. 
[70] A. P. Castano, S. L. Lin, T. Surowy et al., "Serum amyloid P inhibits fibrosis through fc $\gamma \mathrm{R}$-dependent monocytemacrophage regulation in vivo," Science Translational Medicine, vol. 1, no. 5, article 5ra13, 2009.

[71] G. M. Ahrendt, U. S. Tantry, and A. Barbul, "Intra-abdominal sepsis impairs colonic reparative collagen synthesis," American Journal of Surgery, vol. 171, no. 1, pp. 102-108, 1996.

[72] F. J. Thornton, G. M. Ahrendt, M. R. Schaffer, U. S. Tantry, and A. Barbul, "Sepsis impairs anastomotic collagen gene expression and synthesis: a possible role for nitric oxide," The Journal of Surgical Research, vol. 69, no. 1, pp. 81-86, 1997.

[73] S. Khanna, S. Biswas, Y. Shang et al., "Macrophage dysfunction impairs resolution of inflammation in the wounds of diabetic mice," PLoS One, vol. 5, no. 3, article e9539, 2010.

[74] M. E. Swift, A. L. Burns, K. L. Gray, and L. A. DiPietro, “Agerelated alterations in the inflammatory response to dermal injury," The Journal of Investigative Dermatology, vol. 117, no. 5, pp. 1027-1035, 2001.

[75] H. Chen, R. Shi, B. Luo et al., "Macrophage peroxisome proliferator-activated receptor gamma deficiency delays skin wound healing through impairing apoptotic cell clearance in mice," Cell Death \& Disease, vol. 6, no. 1, article e1597, 2015.

[76] R. Mirza, L. A. DiPietro, and T. J. Koh, "Selective and specific macrophage ablation is detrimental to wound healing in mice," The American Journal of Pathology, vol. 175, no. 6, pp. 2454-2462, 2009.

[77] Y. Tang, M. J. Zhang, J. Hellmann, M. Kosuri, A. Bhatnagar, and M. Spite, "Proresolution therapy for the treatment of delayed healing of diabetic wounds," Diabetes, vol. 62, no. 2, pp. 618-627, 2013.

[78] M. O'Reilly, D. E. Newcomb, and D. Remick, "Endotoxin, sepsis, and the primrose path," Shock, vol. 12, no. 6, pp. 411-420, 1999.

[79] M. D. Liang, A. Bagchi, H. S. Warren et al., "Bacterial peptidoglycan-associated lipoprotein: a naturally occurring toll-like receptor 2 agonist that is shed into serum and has synergy with lipopolysaccharide," The Journal of Infectious Diseases, vol. 191, no. 6, pp. 939-948, 2005.

[80] F. Pinheiro da Silva, M. Aloulou, D. Skurnik et al., "CD16 promotes Escherichia coli sepsis through an FcR gamma inhibitory pathway that prevents phagocytosis and facilitates inflammation," Nature Medicine, vol. 13, no. 11, pp. 13681374, 2007.

[81] R. F. Furchgott and J. V. Zawadzki, "The obligatory role of endothelial cells in the relaxation of arterial smooth muscle by acetylcholine," Nature, vol. 288, no. 5789, pp. 373-376, 1980.

[82] L. J. Ignarro, G. M. Buga, K. S. Wood, R. E. Byrns, and G. Chaudhuri, "Endothelium-derived relaxing factor produced and released from artery and vein is nitric oxide," Proceedings of the National Academy of Sciences of the United States of America, vol. 84, no. 24, pp. 9265-9269, 1987.

[83] R. M. J. Palmer, A. G. Ferrige, and S. Moncada, "Nitric oxide release accounts for the biological activity of endotheliumderived relaxing factor," Nature, vol. 327 , no. 6122, pp. 524526, 1987.

[84] M. A. Marletta, P. S. Yoon, R. Iyengar, C. D. Leaf, and J. S. Wishnok, "Macrophage oxidation of L-arginine to nitrite and nitrate: nitric oxide is an intermediate," Biochemistry, vol. 27, no. 24 , pp. $8706-8711,1988$.

[85] C. Nathan, "Nitric oxide as a secretory product of mammalian cells," The FASEB Journal, vol. 6, no. 12, pp. 3051-3064, 1992.

[86] K. Yamasaki, H. D. Edington, C. McClosky et al., "Reversal of impaired wound repair in iNOS-deficient mice by topical adenoviral-mediated iNOS gene transfer," The Journal of Clinical Investigation, vol. 101, no. 5, pp. 967-971, 1998.

[87] Y. C. Hsu, M. Hsiao, L. F. Wang, Y. W. Chien, and W. R. Lee, "Nitric oxide produced by iNOS is associated with collagen synthesis in keloid scar formation," Nitric Oxide, vol. 14, no. 4, pp. 327-334, 2006.

[88] J. E. Park, M. J. Abrams, P. A. Efron, and A. Barbul, "Excessive nitric oxide impairs wound collagen accumulation," The Journal of Surgical Research, vol. 183, no. 1, pp. 487-492, 2013.

[89] R. Ricciardi, P. L. Roberts, P. W. Marcello, J. F. Hall, T. E. Read, and D. J. Schoetz, "Anastomotic leak testing after colorectal resection: what are the data?," Archives of Surgery, vol. 144, no. 5, pp. 407-411, 2009.

[90] A. Vignali, L. Gianotti, M. Braga, G. Radaelli, L. Malvezzi, and V. D. Carlo, "Altered microperfusion at the rectal stump is predictive for rectal anastomotic leak," Diseases of the Colon and Rectum, vol. 43, no. 1, pp. 76-82, 2000.

[91] B. Beck-Schimmer, R. C. Schimmer, C. Madjdpour, J. M. Bonvini, T. Pasch, and P. A. Ward, "Hypoxia mediates increased neutrophil and macrophage adhesiveness to alveolar epithelial cells," American Journal of Respiratory Cell and Molecular Biology, vol. 25, no. 6, pp. 780-787, 2001.

[92] M. C. Bosco, S. Delfino, F. Ferlito et al., "The hypoxic synovial environment regulates expression of vascular endothelial growth factor and osteopontin in juvenile idiopathic arthritis," The Journal of Rheumatology, vol. 36, no. 6, pp. 1318-1329, 2009.

[93] G. Melillo, T. Musso, A. Sica, L. S. Taylor, G. W. Cox, and L. Varesio, "A hypoxia-responsive element mediates a novel pathway of activation of the inducible nitric oxide synthase promoter," The Journal of Experimental Medicine, vol. 182, no. 6, pp. 1683-1693, 1995.

[94] J. V. Dovi, L. K. He, and L. A. DiPietro, "Accelerated wound closure in neutrophil-depleted mice," Journal of Leukocyte Biology, vol. 73, no. 4, pp. 448-455, 2003.

[95] G. F. Pierce, "Inflammation in nonhealing diabetic wounds: the space-time continuum does matter," The American Journal of Pathology, vol. 159, no. 2, pp. 399-403, 2001.

[96] R. G. Reish and E. Eriksson, "Scar treatments: preclinical and clinical studies," Journal of the American College of Surgeons, vol. 206, no. 4, pp. 719-730, 2008. 


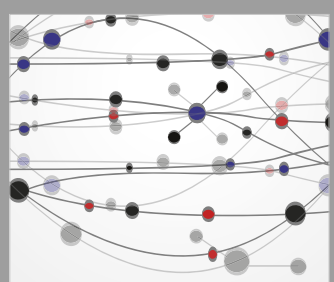

The Scientific World Journal
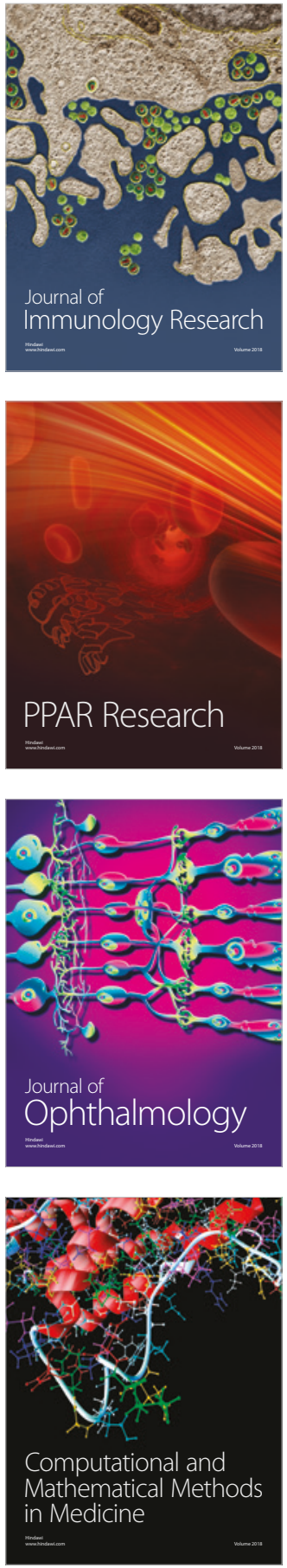

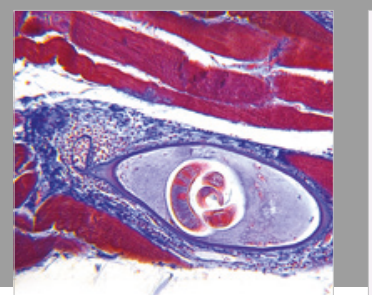

Gastroenterology Research and Practice

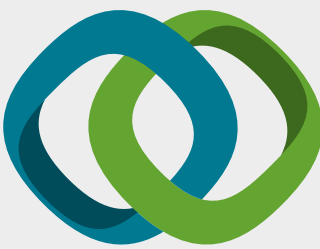

\section{Hindawi}

Submit your manuscripts at

www.hindawi.com
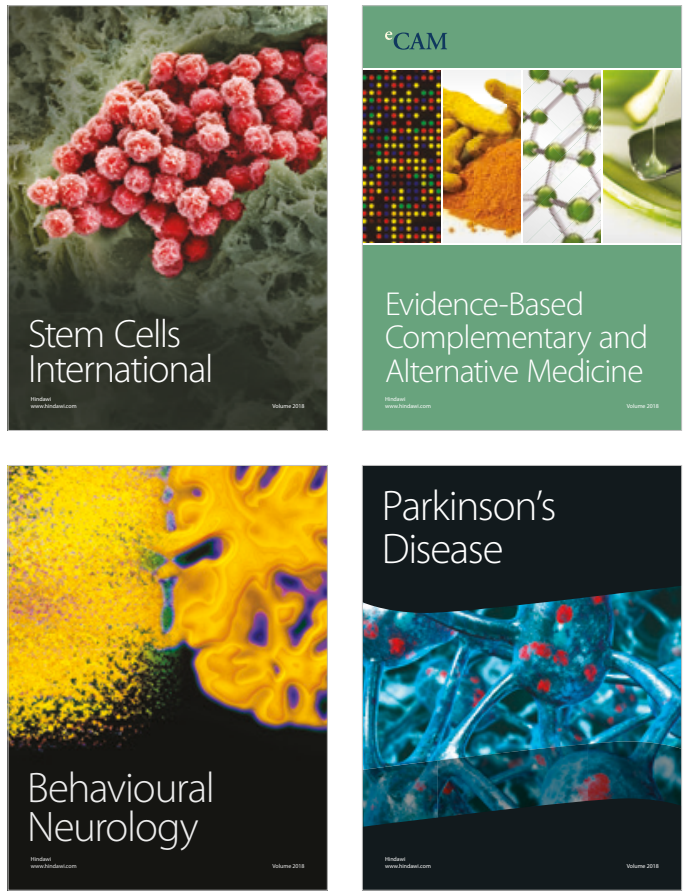

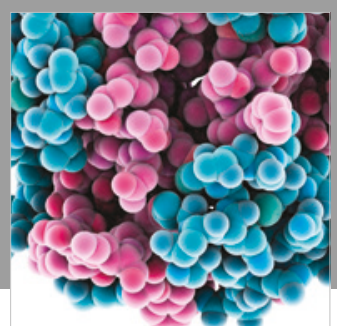

ournal of

Diabetes Research

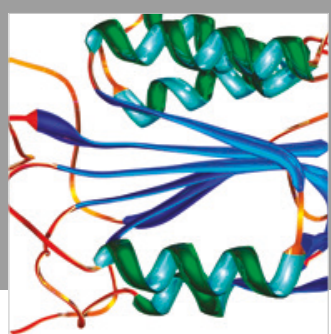

Disease Markers
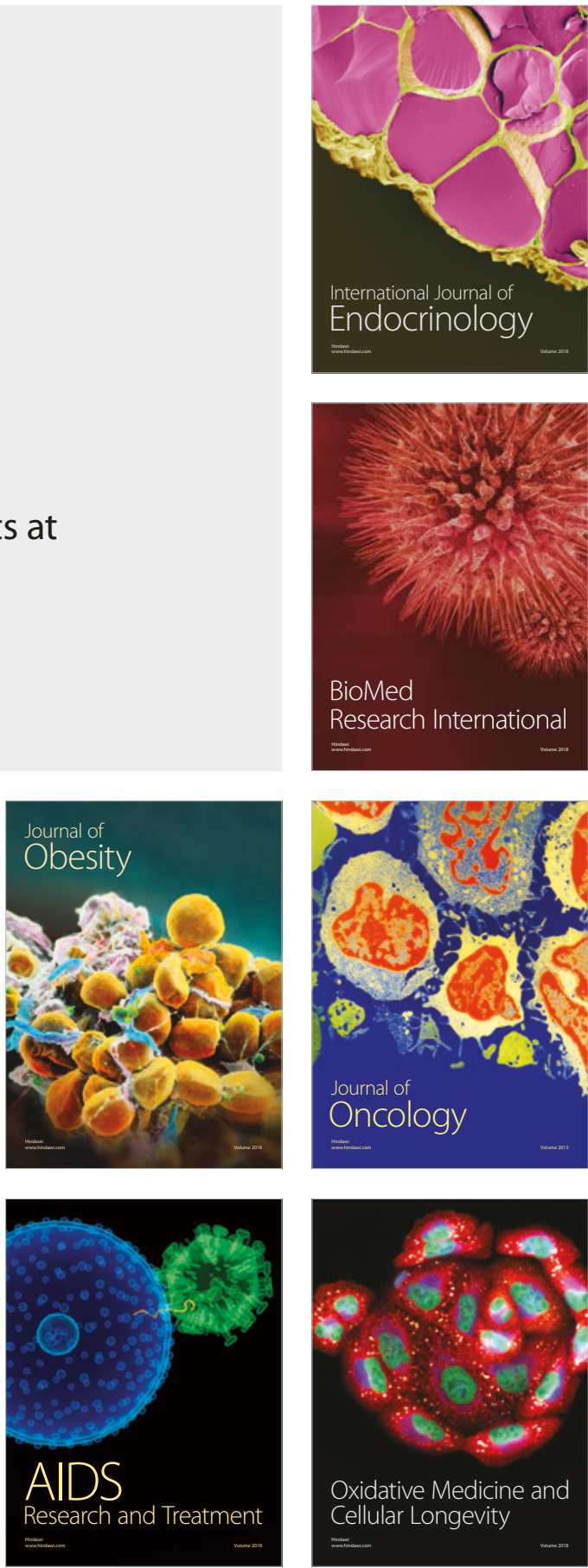